Janos Csorba

Elek Dinya

Paul Plener

Edit Nagy

Eszter Páli
Received: 1 February 2008

Accepted: 4 October 2008

Published online: 22 January 2009
J. Csorba, MD, PhD ( ()

Department of Pathophysiology

Bárczi Gusztáv Faculty of

Special Education

Eotvos Lorand University of Sciences

Budapest, Hungary

E-Mail: janos.csorba@barczi.elte.hu

\section{E. Dinya, $\mathrm{PhD}$}

Medical Department

EGIS Pharmaceuticals PLC

Budapest, Hungary

P. Plener, $\mathrm{MD}, \mathrm{PhD}$

Clinic for Child and Adolescent Psychiatry and Psychotherapy

University of Ulm

Ulm, Germany

E. Nagy, MD · E. Páli, MD

Child and Adolescent Psychiatric Centre

Unified Health Institutions

Pécs, Hungary

\title{
Clinical diagnoses, characteristics of risk behaviour, differences between suicidal and non-suicidal subgroups of Hungarian adolescent outpatients practising self-injury
}

Abstract Objective Self-injury (SI), self-injurious behaviour (SIB), including suicidal or nonsuicidal self-injury (SSI, NSSI) represent an increasing problem among teenagers amounting to a $6-17 \%$ prevalence rate in adolescence, yet little data exists on detailed characteristics and associated factors of SI reaching clinical severity. There is also a scarcity of data distinguishing between suicidal and non-suicidal subsamples of self-injuring patients, i.e. showing which predictors contribute to develop selfinjurious behaviour with a previous suicidal history (SSI). Method Clinical diagnoses and characteristics of risk behaviour were examined in a crosssectional design in suicidal and non-suicidal subgroups of Hungarian adolescent outpatients practising selfinjurious behaviour. From the total pool of 708 new patients consecutively referred with various psychiatric problems in five regional child psychiatric centres in Western-Hungary over an 18month period, 105 adolescent outpatients suffering from selfinjurious behaviour participated in the study (28 males and 77 females aged from 14 to 18 years, mean age 15.97, SD 1.05). The Ottawa/Queen's self-injury questionnaire (OSI) was used to mea- sure the characteristics of risk behaviour, while the comorbid clinical diagnoses were confirmed by the M.I.N.I. Plus International Neuropsychiatric Interview. Descriptive statistics presented the frequencies of the characteristics of SI, bilateral comparisons were used to reveal relevant items to differentiate between sex, duration of practice and SSI versus NSSI and logistic regression was performed to identify significant predictors of suicidal subtype of selfinjuring practice. Results A total of $60 \%$ of the clinical SI population experienced a present or past episode of major depression. The motivation of patients to resist impulses and to discontinue malpractice was low. Cutting and scratching was the most common self-injuring methods. Two-thirds of the sample practised the impulsive type of SI, while $30 \%$ practised premeditated SI having an incubation time from $30 \mathrm{~min}$ to days and weeks before carrying out SI. Although duration of SI did not distinguish the sample in important aspects, girls and boys differed in several aspects of SI practice. SSI adolescents differed from their NSSI peers in a number of important characteristics including the frequency of actions, injured areas, methods, specific stresses and motivations. SSI ado- 
lescents were more likely to favour cutting of the lower leg and drug overdose as modes of SI. SSI adolescents were more likely to report addictive features than their peers with no suicidal motivation. From the aspect of self-injurious practice, logistic regression analysis found only two significant predictors for the combined pathology.

Key words regional-representative Hungarian sample of adoles- cent SI outpatients Ottawa/Queen's self-injury questionnaire (OSI) - M.I.N.I. Plus International Neuropsychiatric Interview - SSI/NSSI comparisons - characteristics of risk behaviour - predictors of SSI

\section{Introduction}

Self-injury (SI), self-injurious behaviour [deliberate self-harm (DSH), parasuicide, self-mutilation, selfcutting, etc., for terminological definitions and synonyms see below] is an important problem in teenagers and creates difficult challenges for treatment in child and adolescent psychiatric centres throughout the world. According to community-based studies, self-injury (SI, SIB) including suicidal and non-suicidal self-injury (SSI, NSSI) is increasingly prevalent in secondary school reports and in community-based surveys of adolescents ranging from 2.2 to $15 \%$ [12, $32,43]$. However, although researchers do not include cases of violent SI in the statistics of DSH in the majority of studies [19-21], frequency data of SI may vary depending on whether impulsive or non-impulsive self-poisoning is considered self-injurious practice by the authors. Hungarian data from the "Child and adolescent self harm in Europe (CASE)" project studying the risk factors of youth suicide in a sample of more than 4,000 adolescents failed to discriminate between suicidal and self-injurious behaviour. The authors presented the frequency of self-harming attempts $7-8 \%$ [15] in a statistically aggregated form containing both suicidal and non-suicidal cases of deliberate self-harming attempts.

Nonfatal deliberate SI or self-harm is not only most common in young people, especially young females $[20,42]$, but is also the most frequent diagnosis among adolescent females in outpatient psychiatric facilities [18, 20, 21, 25]. Ross and Heath [40] stress that $13.9 \%$ of students, especially girls, in community samples report self-mutilation.

\section{Definitions, terms, epidemiology}

Research related to SI faces manifold difficulties in defining the specific character of self-injurious behaviour and in discriminating among similar forms of actions resulting in self-harm. Regarding selfmutilating behaviour (SMB), Ross and Heath stress that "there is no generally agreed upon terminology and a variety of terms have been described in the literature". However, their description is valid not only for the terminological problems regarding self-mutilation, self-laceration, self-carvers [43], selfwounding, wrist/cutting syndrome, delicate self-cutting [41], etc., but more generally refers to interchangeable use of the terms DSH, self-destructive behaviour, self-injurious behaviour (in cases of developmental delay, SIB), SI or even the outdated term "parasuicide". There are inconsistencies in using the term DSH including SI, suicide attempts and overdose cases with mixed motivation in one category.

Self-injury and suicide. Menninger's dynamic point of view from 1938 [31] regarded self-mutilation as a morbid form of self help "to avert total annihilation that is to say suicide". Apart from the fact that he was the first researcher to place self-mutilation in the framework of psychoanalytic theory of defence mechanisms, he presented the first differentiation or contrast between suicide and self-injury.

Pattison and Kahan made a new effort [34] to broaden the definition by defining a "deliberate selfharm syndrome" and proposed rating self-destructive behaviour on a continuum from "low" to "high lethality" and from "direct" to "indirect" self destruction, drawing a distinct line between DSH syndrome as direct low-lethality self-destructive behaviour and that of the suicide attempt as a form of direct self-harm with high lethality. They cited the repetitive character of selfinjurious actions stressing $63 \%$ multiple episodes in a review of case reports. Favazza [13] used the terms major, minor and repetitive types of self-mutilation. Concerning modes of self-harm, skin-cutting and selfmutilation have been found to be the most common forms of SI followed by skin-burning and self-hitting [12].

In 1991, Winchel and Stanley [48] came to a definition proposing that "self-injurious behaviour be defined as the commission of DSH to one's own body. The injury is done to oneself, without the aid of another person, and the injury is severe enough for tissue damage (such as scarring) to result". Acts that are "committed with conscious suicidal intent or are associated with sexual arousal are excluded". However, this conclusion does not consider the addictive, excitement-seeking character of actions. Self-injurious behaviour (SIB) was defined by Pies and Popli [36] as "any intentional act that results in organ or tissue damage to the individual, regardless of moti- 
vation or of "mental state" and without referring to any wish to die". In this definition the differences in motivation between SI and suicide attempts remain obscure. It is to be considered, however, that intention is not prominent in each case. A number of actions occur in a dissociative mental state, i.e. under influence of alcohol/drugs where levels of consciousness and clear motivation to kill oneself are difficult to identify. To make the demarcation line between suicide and SI more characteristic, Petermann and Winkel stated that "self-injurious behaviour is the same as a functionally motivated, direct and open injury or mutilation of one's body, which is not socially accepted and is not accompanied by suicidal ideation." [35]. Meanwhile the term "deliberate selfharm" was widely used in the UK and Australia as a term for adolescents' SI-not only by cutting, but also by ingesting drugs, i.e. impulsive or planned selfpoisoning with substances and without a differentiation between the suicidal or non-suicidal intent of the patient. Skegg [45] tried to clarify the different definitions by collecting terms which are mistakenly used synonymously, from parasuicide to self-wounding. One of the main differences one has to be aware of from the clinical perspective is the issue of distinction between self-injurious and suicidal behaviour. Favazza [14] defined SMB as having no conscious suicidal intent and provided a completed definition [11]: “A culturally not accepted conscious, intentional, repetitive, low-lethality action of damaging one's own body, without suicidal intent". Ross and Heath stress (op. cit.) that the distinction between subgroups of self-harm including suicidal versus nonsuicidal intent is critical. Recently, Muehlenkamp [32] argued with phenomenological and empirical data to suggest that self-injurious behaviour is a distinct disorder for adopting in the next revision of DSM. He referred the obvious lack of conscious suicidal intent or ideation as the first criterion of SI.

Non-suicidal self-injury, the term recently coined by Lloyd-Richardson as a contrast to its counterpart suicidal self-injury (SSI) seems to eradicate the previous terminological confusion [27]. In accordance with this recent viewpoint, the specific terms self-injury, suicidal and non-suicidal self-injury and their acronyms (SI, SSI, NSSI) are used in this study and both the former terminological formulations (parasuicide, self-mutilation, self-carving, etc.) and the term DSH, SIB and several overlaps with suicide and poisonings are intentionally avoided.

Hungary has had the highest suicide rate in the world for over a century prompting remarkable research into suicidal and "deviant" behaviour [i.e. 2, $10,23,37,38]$. The high rate of suicidal and depressed patients both in clinical and community surveys support the feasibility of conducting research into depressive disorders, and forms of suicidal and selfinjurious behaviour in Hungary.

\section{Aims}

The survey aimed to

(a) establish clinical diagnoses as well as provide a basic description of symptoms of SI in the representative clinical sample focusing on the most striking results of the Hungarian survey,

(b) compare characteristics of risk behaviour including motivation, afflicted body areas, modes of SI, affective regulation and addictive aspects of SI between subsamples of the patient population divided by sex, length of malpractice and occurrence of lifetime suicide attempt(s) and

(c) identify independent predictors of the combined (SSI) pathology.

\section{Hypotheses}

Few differences are expected between male and female self-injurers, but more between the SI malpractice of beginners and more practised self-injurers, as chronic SI inflicted over a period of time is expected to have more serious characteristics (frequency, extended body regions, manifold motivation, more complications, addictive features, further treatment, etc.) than that of beginners. Suicidal self-injurers and their nonsuicidal counterparts (according to whether the suicide attempt occurred prior to present SI: SSI vs. NSSI) are expected to differ with regard to the following: depression, comorbid psychiatric disorders, the repetitive or non-repetitive character of the actions, incubation time, the seriousness of the attempts, preferred body regions, motivation and the addictive qualities of their SI. Focusing on developmental variables of double pathology, several predictors of self-injurious items are expected to contribute significantly.

\section{Methods}

\section{Sample}

All the cases were selected from a larger sample of 708 consecutively referred 14 - to 18-year-old outpatients (patients with a new-onset illness who were not diagnosed previously elsewhere) during a 18 month period in five regional child psychiatric facilities in five Transdanubian counties in Hungary referred in 2004-2005 (each county has had one Child and Adolescent Psychiatric Centre in the county capital). 
To be considered for the multicentre project, a child had to meet the following criteria: being neither mentally retarded nor psychotic, showing no evidence of any major medical disorder, having outpatient status and a realistic need for therapy (i.e. purely administrative cases were excluded), living with (a) parent(s) or guardian(s).

Of the registered total pool, 68 refused to fill the test battery (8.5-14\% refusal rate per county centres), while from the eligible test battery of 640 newly treated adolescents 532 had a diagnosis confirmed by the M.I.N.I. Neuropsychiatric Diagnostic Interview. From this group 111 patients had practised recent or past SI within the previous 6 months (ideators were calculated only if thoughts/urges were accompanied by violent self-destructive behaviour). In the sample 21 adolescents had had a concomittant medicament overdose beside their self-injurious violent act(s) but only 6 patients had taken drugs without SI, the latter being excluded from the study. The final sample consisted of 105 patients participating in the study, 28 males and 77 females aged from 14 to 18 years (mean age 16.64 years, SD 1.02 and 16.15 years, SD 1.46, $p=\mathrm{ns})$.

\section{Assessment methods}

(a) To assess risk behaviour, the Hungarian pilot version of the Ottawa/Queen's Self-Injury Questionnaire (OSI, 33) was used. The OSI is widely used to measure forms of SI both in communitybased surveys of adolescents as well as in clinical samples in Canada, Germany and elsewhere in Europe. The OSI is comprised of a series of questions covering many different aspects of SI including the following: urges and acts, feelings and motivation accompanying the behaviour, items assessing the impulsive nature of the behaviour, the time period passing between the impulse and the act of SI, the efficacy of the acts as well as when and how the behaviour started and what factors maintain it. The self-reported effectiveness of SI for regulating negative affects and a scale to measure the motivation to stop engaging in SI are also added. The test stresses the distinction between SI and suicide and has a good test/retest reliability [6]. A Hungarian translation (pilot version) and adaptation was made [7] by the first author and the questionnaire was administered after written consent and parental approval. The procedure was approved by the Scientific Council of the Hungarian Ministry of Health as well as by the Institutional Review Boards of the mother hospitals of the local child psychiatric centres. (b) Clinical diagnoses of the patients were confirmed (79\% in the representative patient pool but $100 \%$ in the self-injuring sample) by the Hungarian standard version of the Mini International Neuropsychiatric Interview (M.I.N.I. Plus). The International M.I.N.I. interview of Sheehan and Lecrubier [44] (Hungarian adaptation by Balazs and Bitter $[4,5])$ contains 18 categorical diagnoses including 4 diseases (major depression, mania, panic disorder, somatisation disorder) with two time frames (rating the current and one past episode in the affective disorders and current/lifetime episodes in the other two). Outside the DSM diagnoses, the M.I.N.I. additionally has a distinct category of "suicidal behaviour" measuring lifetime suicidality listing six items. The items reflect basic symptoms of psychiatric diseases according to DSM-IV. and parallel ICD-10 diagnoses. The adult version was used which can be applied from the age of 14 years. Psychiatric diagnosis were assessed independently from the diagnosis of SIB by the OSI.

\section{Statistical methods}

Basic descriptive statistics were made on each continuous (mean, SD, min, $\max , N$ ) and categorical variable (median, min, $\max , N$ ) and comparisons were made to elicit significant differences between gender and duration of practice and SSI versus NSSI by the Mann-Whitney $U$ test for the non-normal distribution continuous variables and the MaximumLikelihood Pearson Chi-square test for the categorical ones at contingency tables. The most important risk behaviour factors uncovered by previous univariate comparisons were entered into a logistic regression analysis to identify predictors of the SSI practice. Double criteria were used to build the dependent variable SSI: only patients with a verified diagnosis of "suicidal behaviour" by the M.I.N.I. Diagnostic Interview coupled with the confirmed lifetime history of suicidal events measured by the Ottawa Self-Injury Inventory entered the analytic process. The level of significance used was $P<0.05$. All statistical analyses were performed using an SAS/STAT, Software 9.1.3. package.

\section{Results}

Figure 1 presents the most frequent diagnoses of selfinjuring patients using the M.I.N.I. Plus interview. a total of $71 \%$ of the sample had a diagnostic combination of only four diseases: major depression, dys- 
Fig. 1 Diagnoses of SI patients

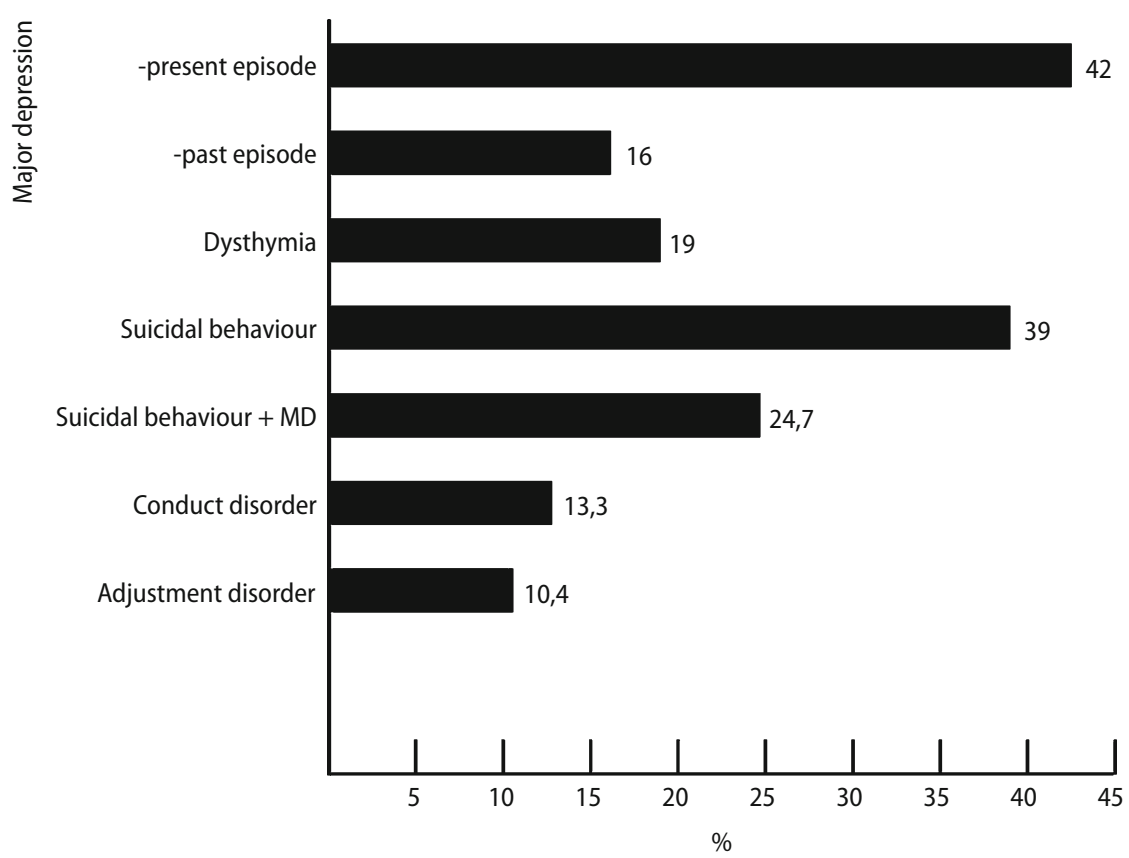

thymia, suicidal behaviour and at least one syndrome from a group encompassing seven anxiety disorder syndromes (panic, agoraphobia, specific phobia, social phobia, GAD or general anxiety disorder, OCD and PTSD). A total of $42 \%$ had an MD present episode and nearly $60 \%$ had either a recent or a past MD episode; 39\% had a diagnosis of "suicidal behaviour" by the M.I.N.I. questionnaire. The comorbidity of suicidal behaviour with MD among the SI patients accounted for $24 \%$, while if dysthymia is also included, the comorbidity of suicidal behaviour with depressive disorders rises up to $32 \%$. From the 105 patients, the majority had multiple clinical diagnoses (60\%). Apart from affective diagnoses, three further disorders accounted for at least 10\%: namely any form of anxiety disorder, conduct disorder and adjustment disorder (26.6, 13.3 and $10.4 \%$, respectively).

From a number of results of descriptive statistics only the most informative items are presented here. $87 \%$ had had lifetime suicidal thoughts, $21 \%$ had suicidal ideas weekly or daily. The qualities of selfdestructive impulses are stressful (41\%), intrusive $(36 \%)$ or comforting $(33.3 \%$ !). Only half the sample inform others before acting, friends being preferred (46.7\% not presented in figures). The peak age for starting self-injurious practices is about 14 (Fig. 2). Concerning the time of duration of SI, $79 \%$ of the patients started it within 1 year, while $20 \%$ are chronic self-injurers (Fig. 3 ).

The most afflicted current regions and those when SI started are presented in Fig. 4, the lower arm, hand with fingers and lower leg being the most preferred

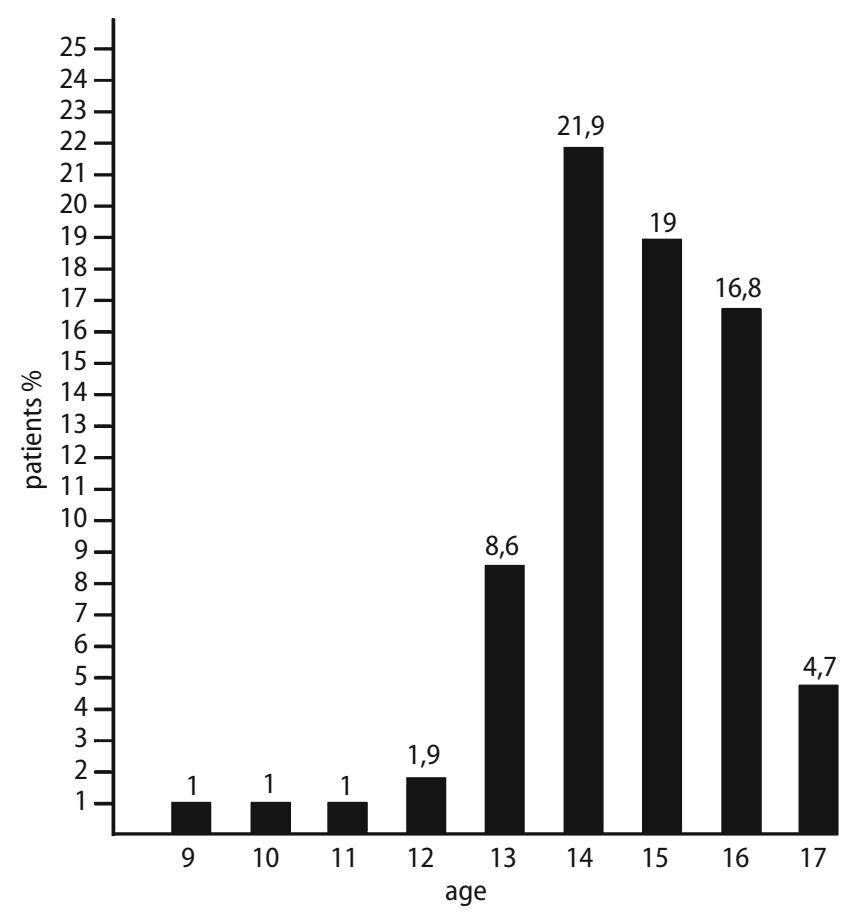

Fig. 2 Age of starting of $S I(n=80)$

regions. The injured body areas appear more frequently with the passing of time.

The methods of SI and emotional causes are presented in Fig. 5. showing that nearly two-thirds of the sample practise self-carving and self-cutting. Of the 20 reasons listed by the Ottawa questionnaire why they started and continue SI, the adolescents ranked "to 
Fig. 3 Duration of SI $(n=79)$

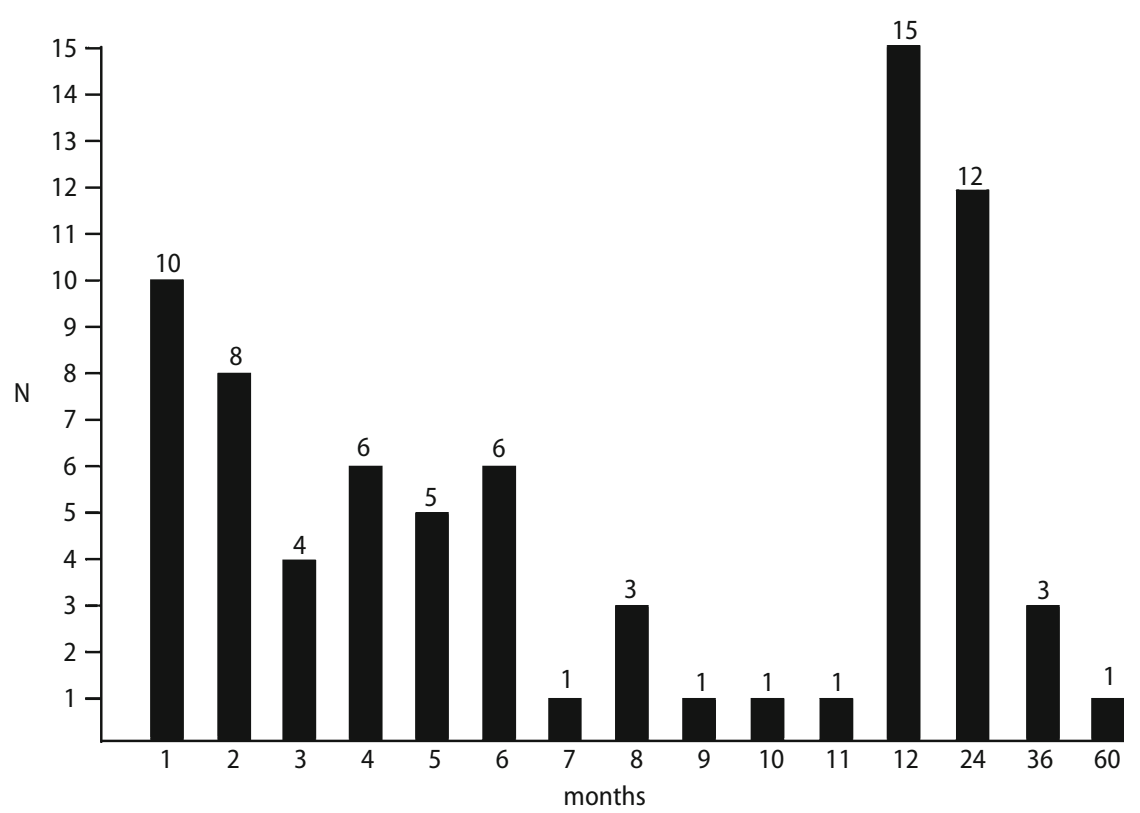

Fig. 4 Injured body areas

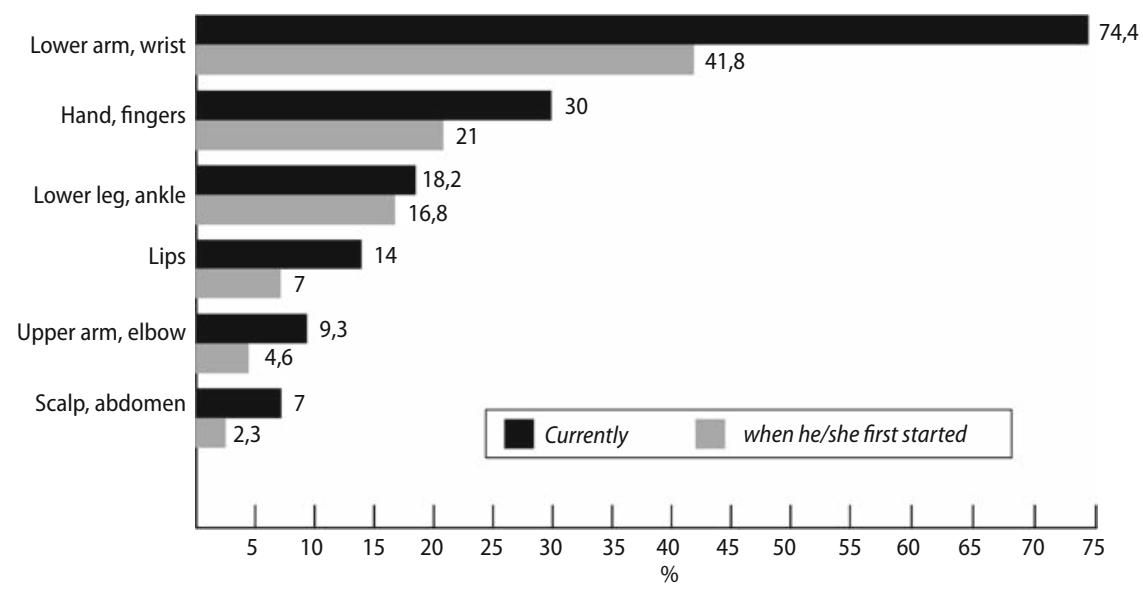

release anger" first on both sites (27 and 18\%, respectively). What is not shown in the figures is that the overwhelming social causes are loss (unspecified $45.7 \%$ ) and failure (23.8\%). Half the sample feel no or only mild physical pain during injury and $35 \%$ of the adolescents feel relief after harming themselves.

The effectiveness of SI was estimated to be only $5-28 \%$ successful in gaining relief from different emotional modalities (tension, anger, anxiety, depression, etc.) on Likert scales. As seen in Fig. 6, the self-injuring adolescent patients seem to fall into two subtypes according to how much time passed between considering and carrying out the action: the ratio is 2:1 between the "impulsive" and "planned" subtypes, the first type taking 1-30 min to perfect the self-injuring act, while the others have an incubation time of prolonged premeditation ranging from half an hour to several days or weeks.

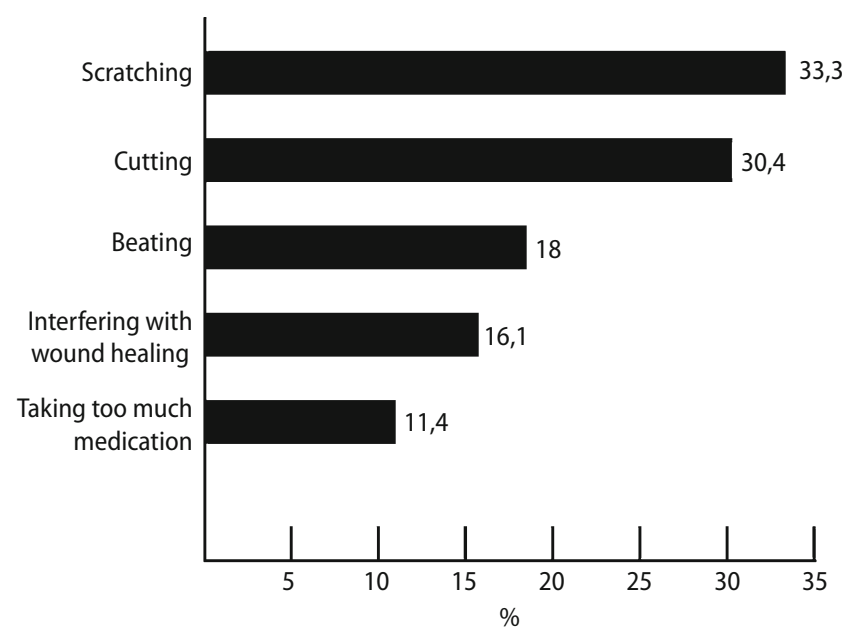

Fig. 5 The most frequent methods of self-injuring 


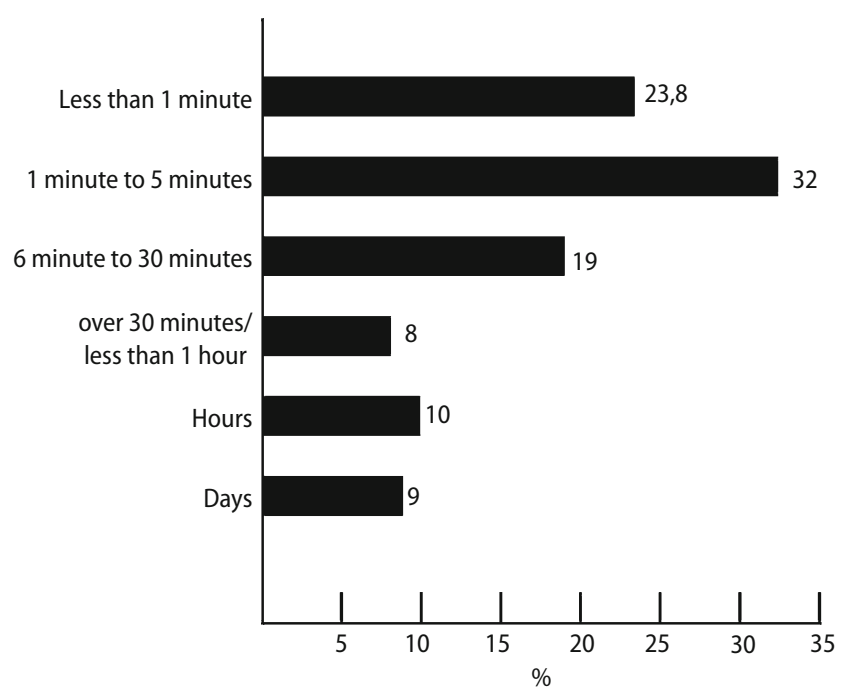

Fig. 6 Time passing between thinking and doing SI

About $10-20 \%$ of the self-injuring adolescents reported addictive features (the self-injurious behaviour occurs more often than intended, the severity has increased, they need to injure themselves more frequently or with greater intensity to produce the same effect, it consumes a significant amount of time, or important social, family, academic or recreational activities were reduced because of the behaviour), but $38 \%$ answered "yes" to the question "Do you continue this behaviour despite recognising that it is harmful to you physically and/or emotionally?".

If trying to resist hurting themselves, only $23.7 \%$ ranked substitute activities like talkig with somebody, reading, writing, music or dancing dance first or second which demonstrates the adolescents' relatively low capacity to avert the self-injurious process. Little more than $40 \%$ are motivated to change or stop the malpractice. More than half received no treatment at all although 11-13\% were admitted to hospital.

Gender differences. Table 1 presents the different characteristics of risk behaviour between boys and girls within the sample, each comparison being taken into consideration only if it accounted for three or more patients. The statistical significance of the following analyses was found using the M-L $\chi^{2}$ statistic set up at $P<0.05$.

Twice as many girls mentioned $\mathrm{SI}^{1}$ to somebody than boys, the latter appearing prone to a greater level of withdrawal than girls. One-third of the girls started SI only 1-2 years ago, while the boys' practice stretched back to 8-9 years. There were significant gender differences in preferred body areas. Regarding areas

\footnotetext{
${ }^{1}$ To avert redundancies, statistical parameters on tables are not repeated in text every time.
}

of the body preferred for SI, a greater proportion of girls than boys ranked the lower arm and wrist, while more boys than girls ranked hands and fingers in the top three, when they first started the malpractice. One and a half or twice as many girls hurt themselves by cutting or scratching than males, while boys bit themselves more frequently than girls. As seen in Table 1, boys were more likely to suffer from the reduction of important recreational events (in family or school, etc.) than girls due to self-cutting practices.

Duration of SI. Beginners and more practised selfinjurers were contrasted regarding the duration of malpractice to reveal possible addictive features among chronic self-injuring adolescents. A total of 26 patients failed to provide data, while $58 \%$ ( 46 of 79 ) of the sample practice SI within 1 year, the median being 7 months. New (<7 months) and old ( $>7$ months) attempters were compared regarding injured body regions and addictive qualities. Unexpectedly, only two differences were found, adolescents with longer SI practice were slightly more addicted in two aspects in their behaviour: increased severity (17 vs. 33\%, $\left.\chi^{2}=3.95, d f=2, P=0.04\right)$ and continuation despite recognition of the disadvantages of SI (30 vs. $53 \%$, $\left.\chi^{2}=7.71, d f=2, P=0.005\right)$, that is two features from seven. No distinct typical injured areas were identified.

Suicidal self-injurious adolescents versus non-suicidal self-injuring peers. Suicidal (SSI) and non-suicidal (NSSI) subsamples of the self-injurious clinical adolescents were contrasted to explore significant differences between the two subtypes. The patients responded to the item "Have you ever made an actual attempt to take your life?", 59 having made no suicide attempt and 46 confirming that they had deliberately tried to take their life earlier. By definition, the patients could not suffer from both SI and suicidal acts at the same cross-sectional point of time, so the questions clearly differentiated between present selfinjuring status and concomittant lifetime suicidal history in $43 \%$ of the sample.

There were no significant differences in age and sex between the two subsamples $\left(\chi^{2}=2.06, d f=1 \mathrm{~ns}\right.$ and $\chi^{2}=3.27, \mathrm{df}=1 \mathrm{~ns}$, respectively). A number of significant differences in risk behaviour were found, the results presenting continuous and categorical variables in the same table (see Table 2).

Regarding clinical diagnosis, we focused on the most relevant disorders appearing previously in the descriptive statistics (major depression, dysthymia, anxiety disorders, conduct disorder and adjustment disorder). As we expected, only major depression differed significantly between the two groups, being more frequent in the SSI groups than among their NSSI counterparts suffering equally from SI. The 
Table 1 Gender differences in risk behaviour

\begin{tabular}{|c|c|c|c|c|c|}
\hline OSI items & $\begin{array}{l}\text { Females } \\
N=77(\%)\end{array}$ & $\begin{array}{l}\text { Males } \\
N=28(\%)\end{array}$ & $d f$ & $\chi$ & $P$ \\
\hline Let some people know that they hurt themselves & $33(43)$ & $6(21)$ & 1 & 4.27 & 0.03 \\
\hline $\begin{array}{l}1-2 \text { years since SI started } \\
\text { Inflicted areas ranked top three }\end{array}$ & $24(31)$ & $4(14)$ & 6 & 13.46 & 0.03 \\
\hline Lower arm/wrist & $39(50)$ & $6(21)$ & 9 & 22.52 & 0.007 \\
\hline Hand/fingers & $6(7)$ & $6(21)$ & & & \\
\hline Injury by cutting & $42(54)$ & $8(28)$ & 1 & 5.71 & 0.01 \\
\hline By scratching & $43(55)$ & $9(32)$ & 1 & 4.69 & 0.03 \\
\hline By biting & $11(14)$ & $9(32)$ & 1 & 3.92 & 0.04 \\
\hline Reduction of important recreational events & $9(11)$ & $8(28)$ & 1 & 5.26 & 0.02 \\
\hline
\end{tabular}

${ }^{\mathrm{a} M-L}$ Pearson Chi-square statistic

Table 2 Non-suicidal (NSSI) and suicidal (SSI) groups of self-injuring adolescents ${ }^{\mathrm{a}}$

\begin{tabular}{|c|c|c|c|c|c|}
\hline OSI risk items & NSSI group $N=59(\%)$ & SSI group $N=46(\%)$ & $\chi^{2}$ & $d f$ & $P$ \\
\hline Clinical diagnosis: major depression ${ }^{\dagger}$ & $22(37)$ & $32(69)$ & 11.51 & 2 & 0.003 \\
\hline Self-injurious practice without the intention to kill him/herself & $M(S D)^{b}$ & $M(\mathrm{SD})$ & $u$ & & \\
\hline Self-injurious thought $/ 6$ months* & $3.00(1.06)$ & $3.29(1.09)$ & 1,043 & & ns \\
\hline Self-injurious thought/1 month* & $2.06(0.99)$ & $2.53(1.06)$ & 2,692 & & 0.002 \\
\hline Actual self-injury/6 month* & $2.51(0.75)$ & $3.06(0.99)$ & 2,866 & & 0.0002 \\
\hline Actual self-injury/1 month* & $1.67(0.80)$ & $2.28(0.92)$ & 2,564 & & 0.001 \\
\hline Suicidal thought-lifetime* & $2.95(1.45)$ & $4.37(1.29)$ & 3,135 & & 0.0000 \\
\hline Let only some people know, that they hurt themselves & $17(28)$ & $22(47)$ & 4.00 & 1 & 0.04 \\
\hline Injured area: lower leg/ankle & $4(6)$ & $10(21)$ & 5.03 & 1 & 0.02 \\
\hline \multicolumn{6}{|l|}{ Method } \\
\hline Cutting & $22(37)$ & $28(60)$ & 5.80 & 1 & 0.01 \\
\hline Severe nail-biting & $3(5)$ & $11(23)$ & 8.13 & 1 & 0.004 \\
\hline Medication overdose & $7(11)$ & $14(30)$ & 5.57 & 1 & 0.01 \\
\hline \multicolumn{6}{|l|}{ The reason for starting is } \\
\hline To relieve nervousness/fearfullness & $12(20)$ & $17(36)$ & 3.55 & 1 & 0.05 \\
\hline To stop feeling alone and empty & $5(8)$ & $11(23)$ & 4.77 & 1 & 0.02 \\
\hline \multicolumn{6}{|l|}{ Specific stress affecting self-injury } \\
\hline Abandonment (friend, parent) & $20(33)$ & $25(54)$ & 4.42 & 1 & 0.03 \\
\hline \multicolumn{6}{|l|}{ Addictive features } \\
\hline SI occurs more often than intended & $3(5)$ & $19(41)$ & 23.47 & 2 & 0.0001 \\
\hline The severity of self-injurious behaviour increased & $8(13)$ & $14(30)$ & 7.15 & 2 & 0.02 \\
\hline The need to injure themselves with greater intensity to produce the same effect & $6(10)$ & $15(32)$ & 11.50 & 2 & 0.003 \\
\hline Important social activities reduced & $6(10)$ & $11(23)$ & 8.83 & 2 & 0.01 \\
\hline Trying to resist, they talk to someone & $14(23)$ & $19(41)$ & 3.69 & 1 & 0.05 \\
\hline
\end{tabular}

${ }^{\mathrm{a} M-L}$ Pearson Chi-square statistics

* Mann-Whitney $U$ test

${ }^{\mathrm{b}}$ Mean, SD

†No OSI item

occurrence of conduct disorder did not differ between the SSI and NSSI groups ${ }^{2}$.

Self-injuring adolescents having a lifetime suicidal history (at least one suicidal act reported during the patient's lifetime) rated more suicidal thoughts according to expectations, but have a more serious recent SI history compared with their NSSI peers, having more self-injurious ideas and actions within the last half year. SSI adolescents share their actions with people less than their counterparts (Table 2).

\footnotetext{
${ }^{2}$ Other diagnoses reaching no significant differences are not presented in the table.
}

SSI adolescents were more likely to favour cutting/ scarification of the lower leg, severe nail-injury and drug overdose as modes of SI. They were twice or even four times more likely to use SI to avert fearfullness and feelings of emptiness than their NSSI peers. They suffer more frequently from abandonment and refusal of their personality comparing the same parameters with non-suicidal self-injuring adolescents and are clearly more addictive to SI than NSSI patients (frequency, severity, intensity, cut-off from important social events). SSI adolescents were more likely to use talking as a way of resisting method against SI than their non-suicidal self-injuring peers. Despite expectations, there were similar degrees of repetitiveness in 
Table 3 Independent effects of variables on suicidal/self-injurious (SSI) behaviour ${ }^{\mathrm{a}}$ (logit Chi-square (5) $12.76, P=0.032$ )

\begin{tabular}{|c|c|c|c|}
\hline & $\mathrm{OR}$ & $\mathrm{Cl} 95 \%$ & $\mathrm{P}$ \\
\hline Const. BO & 0.008 & -9.79 to 0.35 & ns \\
\hline Injured area: lower leg, ankle & 22.20 & 0.26 to 5.93 & 0.026 \\
\hline Medication overdose & 4.467 & -1.21 to 4.21 & 0.261 \\
\hline They now need to injure themselves more frequently or with greater intensity to produce the same effect & 31.03 & 0.16 to 6.70 & 0.032 \\
\hline Self-injurious behaviour or thinking about it consumes a significant amount of time & 0.438 & -3.63 to 1.98 & 0.554 \\
\hline They continue this behaviour despite recognising that it is harmful physically and/or emotionally & 0.321 & -3.88 to 1.62 & 0.408 \\
\hline
\end{tabular}

aLogistic regression analysis, confounders are controlled. Significant effects are marked in italics

both subsamples and non-suicidal self-injurers did not practice injury more frequently than their suicidal counterparts.

Logistic regression analyses were performed to estimate the relationships between dependent suicidal/self-injury (SSI) and its correlates allowing for the determination of the independent effects of the characteristics of risk behaviour in the presence of the effects of other variables.

The dependent variables of logits were (a) the M.I.N.I.diagnosis of suicidal behaviour, (b) lifetime suicide attempt(s) detected by OSI, and (c) a combination of the above in the first, second and third analysis, respectively. Regarding the sensitivity and specificity of the models, the combined last version provided the best ratio of correctly classified cases (59.9 and $86.8 \%$, Odds ratio 13.5 , percentage correct $75.8 \%)$ and this version was perfected. The main effects had to have met an entry criteria of $P>0.10$ to be retained in the model. After a series of semipartial correlations of the 52 behaviour items, 5 entered the logit process adjusted for age and sex. One area item, one method item and three addictive features were included in the analysis. As seen in Table 3, only 2 variables proved to be significant in the process but with strong odd ratios, (a) preference for the lower leg/ankle region (OR 22.20, CI 95\% 0.26-5.93, $P=0.003)$ and (b) one addictive item "if the selfinjury produced an effect when started, do you now need to self-injure more frequently or with greater intensity to produce the same effect?"(OR 31.03, CI 95\% 0.16-6.70, $P=0.04$ ).

Unexpectedly, we found no further significant independent contributions of specific behavioural items such as the frequency of self-injurious thought, other involved body regions and methods possibly typical for suicide attempts, i.e. medicament overdose, specific emotional causes and further addictive qualities.

\section{Discussion}

When interpreting our results, we must take into consideration that our sample is drawn from a clinical adolescent outpatient population, data and conclusions for whom presumably differ from those of recruited from studies on adolescent inpatients or from school surveys.

Depression is the most common diagnosis. Like other researchers [32, 49], we had a predominance of girls in our clinical SI population including only new cases, yet the rate seems equalized by aging, as there are some reports [46] as to the equal prevalence of self-injuries among men and women in adult psychiatric samples. Major depression (with present and past episodes) was found to be the most frequent diagnosis in adolescent outpatients followed by dysthymia and anxiety disorders. A number of authors argue for the importance and high occurrence of depressive moods in adolescent SI patients $[16,17,28$, 34] reporting $45 \%$ depressed patients in adult selfmutilators, while others (i.e. [47]) found depressed moods and heightened anxiety in an adolescent sample. Stanley et al. (op. cit.) found higher levels of depression in those suicide attempters who had a history of self-mutilation compared with a control suicidal group. In the study of Haavisto et al. [18] selfreported depressive symptoms at the age of eight predicted acts of DSH 10 years later. One of the few exceptions is the study of suicide attempters by Langbehn and Pfohl [24] where mutilators were less likely to have diagnoses of major depression than non-mutilating suicidal peers. The relationships between SI with depression and mood disregulation is shown in detail by Nixon et al. [33]. It seems that any form of affective disorder is of eminent importance in self-injuring adolescents.

Conduct disorder, i.e. externalising pathology was less pronounced in the Hungarian sample (10\%). Ayton et al. (op. cit.) differentiated self-harming adolescents from suicidal peers concluding that SI children exhibited more disruptive behaviour and externalising symptoms and had a history of abuse while their suicidal peers suffered from depression displaying internalising features and had a more serious medical condition. There is evidence indicating increased aggression and behavioural problems among both self-injuring adults and adolescents [3, 9, $25,28-30]$, the incidence rate seeming to be depen- 
dent on the recruitment of the sample (samples from a foster care placement include understandably more patients with behavioural problems). Explaining the relative underrepresentation of externalising disorders Guertin (op. cit.) comments on a possible "alternative outlet for the emotions" of the selfinjuring patients practising self-harm rather than displaying behavioural symptoms.

Concerning other comorbid disorders, having only three patients with anorexia nervosa in our study sample allows for no satisfying comparison to be made with results of other studies $[8,12]$ which report $50-60 \%$ of SI patients having an eating disorder. Drug abuse was also underrepresented in our Hungarian sample (6 cases, 3 having a comorbid alcohol abuse too), as adolescent patients suffering from eating disorders or drug problems are cared for paediatric and addictological centres in Western-Hungary beyond the domain of child psychiatric facilities, which may be the main reason for the low number of patients.

Guertin et al. (op. cit.) found a more colourful (though no more serious if seriousness is associated with higher lethality) pathology in adolescents suffering from both suicidal overdose and self-mutilation. Suicidal self-poisoners practising self-mutilation were significantly more likely to be diagnosed with oppositional defiant disorder, major depression and dysthymia, besides which they had higher scores on hopelessness, loneliness, anger, risk taking, recklessness and alcohol abuse than did non-mutilating suicidal peer patients. Stanley et al. (op. cit.) argue that double pathology results in a more serious psychopathological status: they reported SSI patients as having more persistent suicidal ideation and having been more depressed, anxious and impulsive than their suicidal counterparts with no history of selfmutilative practices.

Cutting/scratching was the most common method. The majority of our self-injurig adolescents were selfmutilators. The finding and other results are in accordance with the findings of related literature in many aspects: the age range of the patients [12], the preferred body regions being the arms/elbow/hands/ legs/chest, which is similar to data drawn from a community sample of adolescents [27].

Although there is a consensus among researchers that SI is of a mostly impulsive character [1, 20], Favazza mentioned [14] that an individual may brood on the SI for hours and days engaging in rituals around the act. While we failed to find other comprehensive literature data concerning how much time passes between thinking and carrying out SI, our findings confirm that a third of the self-injurers-similar to those attempting suicide-premeditate for hours or days before the self-injuring action.
Present SI and suicide in the anamnestic data. Although Lloyd [26] reported only a few patients having both suicidal intent and self-cutting behaviour, the SSI patients account for $40-50 \%$ of the SI population in studies (Guertin et al., op. cit.) [22, 47]. In our study $43 \%$ have a history of suicide attempts. Drug overdose seems to play significant role as an accompanying method in the SI practice of those patients who had lifetime suicide attempts.

Adolescent SI patients show many "addictive" features (see Nixon and Cloutier, op. cit.) and it is more pronounced in the SSI subsample. The patient regularly considers SI after a stressful event, the behaviour occuring more often/its repetitive character/and/or its severity increasing, and the patients are unable to resist, they continue SI despite recognising it as harmful, the practice having a comforting quality, accompanied by excitement, yet creating social problems for them, etc. These data underlines the character of self injury as a process.

SI was expected to cease/relieve depressive feelings at first, but other psychological qualities (anxiousness, emptiness, aloneness, abandonment) emerged as having differing importance between the two samples in the Hungarian study. Although diagnosed as depressed, self-injuring youth may feel that other mental representations are more intrusive and painful than sadness and try to cope with them by means of SI. Some authors found no substantial differences among the typical psychological experiences before or after the actions in NSSI adolescents in community-based surveys (Lloyd-Richardson et al., op. cit.). However, a number of authors $[20,39]$ have identified differences regarding the motivational aspects between male and female self-injurers in school and clinical studies.

There were few sex and age differences. No substantial differences were found related to motivation, boys seeming to suffer more from the social disadvantages of self-injuring acts than girls.

We found evidence confirming the increased severity of SI among chronic patients, but no other typical differences were identified between new and older attempters related to features of the malpractice. Logistic regression failed to add any new insights to the univariate analyses, and no characteristic predictors were identified to establish the practice of SSI.

Klonsky and Muehlenkamp (op. cit.) are presumably right in stating that "the link between suicidal and non-suicidal self-injurious behaviour is nuanced". Though we were managed to identify some differences, it is rather difficult to find distinguishing points between the NNSI and SSI subsamples of selfinjurious patients referring to the present characteristics of their malpractice. Further research is needed to identify the differences using community-based and clinical surveys. 
Limitations. The survey had a specific (regional, outpatient, clinical) sample population not representative of every group of SI patients. Because of the fluctuating willingness of the patients to respond, the case numbers of comparison cells varied remarkably. Our cross-sectional study enables more limited implications than a longitudinal one.
Acknowledgments We would like to thank the following Hungarian colleagues from the regional child psychiatric centres participating in the Pannonia study: Zs. Fekete MD, B. Ficsor MD, M. Vados MD. and M. Solymossy (Szekesfehervar), M. Palaczky MD, A. Horvath MD, and A. Bona (Pecs), Zs. Sörfőző MD (Kaposvar), P. Steiner MD and E. Ferencz PhD (Veszprém), E. Harkány MD and Zs. Babrik MD (Zalaegerszeg).

\section{References}

1. Apter A, Plutchik R, van Praag HM (1993) Anxiety impulsiveness and depressed mood in relation to suicidal and violent behaviour. Acta Psychiat Scand 87:1-5

2. Arato M, Demeter E, Rihmer Z, Somogyi E (1988) Retrospective psychiatric assessment of 200 suicides in Budapest. Acta Psychiatrica Scand 77:54-456

3. Ayton A, Rasool H, Cottvell D (2003) Deliberate self-harm in children and adolescents: association with social depression. Eur Child Adolesc Psychiatry 42(6):303-307

4. Balázs J, Bitter I (1998) Elaboration of Hungarian version of M.I.N.I and M.I.N.I Plus diagnostic interview. Psychiatr Hung 13(2):160-168 (Hung.)

5. Balázs J, Bitter I (2000) Criterionvalidity test of M.I.N.I Plus diagnostic questionnaire. Psychiatr Hung 15(2):134-144 (Hung.)

6. Cloutier PF, Nixon MK (2005) The Ottawa self-injury inventory: functional analysis of self-injurious behaviour in adolescents. In: Joint annual meeting of the American and Canadian academies of child and adolescent psychiatry, abstract

7. Csorba J, Ferencz E, Steiner P, Nemeth A (2006) Characteristics of Hungarian adolescents with self-injurious behavior. Results of a pilot study. Eur Psychiatry 21(suppl 1):156-157

8. Darche MA (1990) Psychological factors differentiating self-mutilating and non-self-mutilating adolescent inpatient females. Psychiatr Hosp 21:31-35

9. D’Eramo KS, Prinstein MJ, Freeman J, Grapentine WL, Spirito A (2004) Psychiatric diagnoses and comorbidity in relation to suicidal behaviour among psychiatrically hospitalized adolescents. Child Psychiatry Hum Dev 35(1): 21-35

10. Diekstra RFW (1989) Suicide and the attempted suicide. An international perspective. Acta Psychiatr Scand 78(354):1-24

11. Favazza AR, Rosenthal RJ (1993) Diagnostic issues in self-mutilation. Hosp Commun Psychiatry 44:134-140
12. Favazza AR, Conterio K (1989) Female habitual self-mutilators. Acta Psychiatr Scand 79:283-289

13. Favazza AR, Simeon D (1995) Selfmutilation. In: Hollander E, Stein DJ (eds) Impulsivity and agression. Wiley, New York, pp 185-200

14. Favazza AR (1999) Self-mutilation. In: Jacobs DG (ed) The Harvard Medical School guide to suicide assessment and intervention. Jossey-Bass, San Francisco, pp 125-145

15. Fekete $S$, Hewitt $A$, Vörös V, Osváth $P$ (2004) Factors of suicidal behavior in adolescents on the basis of data of Hungarian centrum (Pecs) of European multicenter study CASE (Hung.). Psychiatr Hung 19(4):337-345

16. Ghaziuddin M, Tsai L, Nylor M, Ghaziuddin N (1992) Mood disorder in a group of self-cutting adolescents. Acta Paedopsychiatr 55:103-105

17. Guertin T, Lloyd-Richardson E, Spirito A, Donaldson D, Boergers J (2001) Selfmutilative behavior in adolescents who attempt suicide by overdose. Am Acad Child Adolesc Psychiatry 40(9):10621069

18. Haavisto A, Sourander A, Multimaki P, Parkkola K, Santalahti P, Helenius H, Nikolakaros G, Moilanen I, Kumpulainen $\mathrm{K}$, Piha J, Aronen E, Puura K, Linna SL, Almquist F (2005) Factors associated with ideations and acts of deliberate self-harm among 18-year-old boys. Soc Psychiat Psychiat Epid 40(11):912-921

19. Hawton K, Cole D (1982) Motivational aspects of deliberate self-poisoning in adolescents. Br J Psychiatry 141:286291

20. Hawton K, Fagg J, Simkin S, Bale E, Bond A (1997) Trends in deliberate self-harm in adolescents in Oxford, 1985-1995. J Adolesc 23:47-55

21. Hawton K, Rodham K, Evans E, Weatherall R (2002) Deliberate selfharm in adolescents: a self-report survey in schools in England. Br Med J 325:1207-1211

22. Klonsky ED, Muehlenkamp JJ (2007) Self-injury: a research review for the practitioner. J Clin Psychol 563(11): 1045-1056
23. Kopp M, Skrabski A, Magyar I (1987) Neurotics at risk and suicidal behaviour in the Hungarian population. Acta Psychiatr Scand 76(suppl 835):2-8

24. Langbehn DR, Pfohl B (1993) Clinical correlates of self-mutilation among psychiatric inpatients. Ann Clin Psychiatry 5(1):45-51

25. Laye-Gindhu A, Schonert-Reichl KA (2005) Nonsuicidal self-harm among community adolescents: understanding the "Whats" and "Whys" of self-harm. J Youth Adolesc 34(5):447-457

26. Lloyd EE, Kelley ML, Hope T (1997) Self mutilation in a community sample of adolescents: descriptive characteristics and provisional prevalence rates. In: Poster, Ann. Meet. Soc. Behav. Med., New Orleans

27. Lloyd-Richardson EE, Perrine N, Dierker L, Kelley ML (2007) Characteristics and functions of non-suicidal self-injury in a community sample of adolescents. Psychol Med 4:1-10

28. Matsumoto T, Azekawa T, Yamaguchi A, Asami T, Iseki E (2004) Habitual self-mutilation in Japan. Psychiatry Clin Neurosci 58(2):191-198

29. Matsumoto T, Yamaguchi A, Chiba Y, Asami T, Iseki E, Hirayashu Y (2005) Self-burning vs self-cutting:patterns and implications of self-mutilation: a preliminary study of differences between self-cuting and self-burning in a Japanese juvenile detention center. Psychiatry Clin Neurosci 59:62-69

30. Matsumoto T, Yamaguchi A, Asami T, Okada T, Yoshikawa K, Hirayashu Y (2005) Characteristics of self-cutters among male inmates:association with bulimia and dissociation. Psychiatry Clin Neurosci 59:319-326

31. Menninger KA (1938) Man against himself. Hartcourt Brace, New York

32. Muehlenkamp JJ (2005) Self-injurious behaviour as a separate clinical syndrome. Am J Orthopsychiatry 75(2):324-333

33. Nixon MK, Cloutier PF, Aggrawal S (2002) Affect regulation and addictive aspects of repetitive self-injury in hospitalized adolescents. J Am Acad Child Adolesc Psychiatry 41(11):1333-1341 
34. Pattison EM, Kahan J (1983) The deliberate self-harm syndrome. Am J Psychiatry 140:867-872

35. Petermann F, Winkel S (2007) Selbstverletzendes Verhalten: Diagnostik und psychotherapeutische. Ansätze Zeitschrift für Psychiatrie. Psychologie und Psychotherapie 55:123-133

36. Pies RW, Popli AP (1995) Self-injurious behavior: pathophysiology and implications for treatment. J Clin Psychiatry 56(12):580-588

37. Rihmer Z (1996) Relationship between recognised depression and suicide in Hungary. Int J Methods Psychiatr Res 6:15-20

38. Rihmer Z (2006) Suicide prevention. In: Akiskal HS, Tohen M (eds) Bipolar psycho-pharmacotherapy caring for the patient. Wiley, Chichester, pp $353-$ 365

39. Rodham K, Hawton K, Evans EB (2004) Reasons for deliberate self-harm: comparison of self-poisoners and self-cut- ters in a community sample of adolescents. J Am Acad Child Adolesc Psychiatry 43(1):80-87

40. Ross S, Heath N (2002) A study of the frequency of self-mutilation in a community sample of adolescents. J Youth Adolesc 31:67-77

41. Rosenthal RJ, Rinzler C, Walsh R, Klausner E (1972) Wrist-cuting syndrome: the meaning of a gesture. Am J Psychiatry 128:1363-1368

42. Schmidtke A, Bille Brahe U, De Leo D (1996) Attempted suicide in Europe: rates, trends and sociodemographic characteristics of suicide attempters during the period 1989-1992. Results of the WHO/EURO Multicentre Study on Parasuicide. Acta Psychiatr Scand 93:327-338

43. Schwartz RH, Cohen P, Hoffmann NG, Meeks JE (1989) Self-harm behaviours (carving) in female adolescent drug abusers. Clin Pediatr 28:340-346
44. Sheehan D, Lecrubier Y (1997) The M.I.N.I. international neuropsychiatric interview: a short diagnostic interview: reliability and validity according to the CIDI. Eur J Psychiatry 12P:224-231

45. Skegg K (2005) Self-harm. Lancet 366(9495):1471-1483

46. Stanley B, Gameroff MJ, Michalsen V, Mann J (2001) Are suicide attempters who self-mutilate a unique population? Am J Psychiatry 158:427-432

47. Whitlock J, Know KL (2007) The relationship between self-injurious behaviour and suicide in a young adult population. Arch Pediatr Adolesc Med 161(7):634-640

48. Winchel RM, Stanley M (1991) Selfinjurious behaviour: a review of the behaviour and biology of self-mutilation. Am J Psychiatry 148:306-317

49. Zlotnick C, Donaldson D, Spirito A, Pearlstein T (1997) Affect regulation and suicide attempts in adolescent inpatients. JAACAP 36:793-798 